AperTO - Archivio Istituzionale Open Access dell'Università di Torino

\title{
LIN-28B/let-7a/IGF-II axis molecular subtypes are associated with epithelial ovarian cancer prognosis
}

\section{This is the author's manuscript}

Original Citation:

Availability:

This version is available http://hdl.handle.net/2318/1599911

since 2016-10-09T12:11:06Z

Published version:

DOI:10.1016/j.ygyno.2015.12.035

Terms of use:

Open Access

Anyone can freely access the full text of works made available as "Open Access". Works made available under a Creative Commons license can be used according to the terms and conditions of said license. Use of all other works requires consent of the right holder (author or publisher) if not exempted from copyright protection by the applicable law. 


\title{
LIN-28B/let-7a/IGF-II axis molecular subtypes are associated with epithelial ovarian cancer prognosis
}

\author{
Lingeng Lu ${ }^{\mathrm{a}, *}$, Dionyssios Katsaros ${ }^{\mathrm{b}}$, Emilie Marion Canuto ${ }^{\mathrm{b}}$, Nicoletta Biglia ${ }^{\mathrm{c}}$, Harvey A. Risch ${ }^{\mathrm{a}}$, Herbert Yu ${ }^{\mathrm{d}}$ \\ a Department of Chronic Disease Epidemiology, School of Public Health, School of Medicine, Yale Cancer Center, Yale University, New Haven, CT 06520-8034, USA \\ ${ }^{\mathrm{b}}$ Department of Surgical Science, A O Città della Salute e della Scienza di Torino, S.Anna Hospital, 10126 Turin, Italy \\ c Department of Surgical Science, Division of Obstetrics and Gynecology, University of Torino School of Medicine, Mauriziano Hospital, 10128 Turin, Italy \\ d Cancer Epidemiology Program, University of Hawaii Cancer Center, Honolulu, HI 96813, USA
}

\section{H I G H L I G H T S}

- Both $L I N-28 B^{\text {high }}$, and $L I N-28 B^{\text {low }} /$ let-7a $a^{\text {high }} / I G F-I I^{\text {high }}$ signatures had high risks of relapse and overall mortality in EOC.

- EOC patients with the $L I N-28 B^{\text {low }} / l e t-7 a^{\text {low }}$ pattern had better response to chemotherapy.

- Four molecular subtypes were classified for EOC patients based on LIN-28B/let-7a/IGF-II axis.

\section{A R T I C L E I N F O}

\section{Article history:}

Received 18 August 2015

Received in revised form 4 December 2015

Accepted 30 December 2015

Available online 2 January 2016

\section{Keywords:}

Epithelial ovarian cancer

LIN-28B/let-7a/IGF-II axis

Molecular subtype

Prognosis

\section{A B S T R A C T}

Objectives. Aberrant expressions of $L I N-28 B$, let-7a and IGF-II occur in epithelial ovarian cancer, and the LIN-28B/let-7a/IGF-II axis is associated with human disease. The purpose of this study was to investigate the associations between $L I N-28 B /$ let-7a/IGF-II axis molecular subtypes and epithelial ovarian cancer prognosis.

Methods. Using quantitative reverse transcription PCR, we analyzed LIN-28B, let-7a and IGF-II mRNA in 211 primary epithelial ovarian cancer tissues, and also performed Classification and Regression Tree (CART) and survival analyses.

Results. Four terminal subtypes were identified in the CART analysis in combination with survival analysis. Kaplan-Meier survival curves showed that subtypes LIN-28B ${ }^{\text {low }} l e t-7 a^{\text {low }}$ and LIN-28B ${ }^{\text {low }}$ let-7 $a^{\text {high }}$ IGF-II ${ }^{\text {low }}$ had significantly better survival than subtypes $L I N-28 B^{\text {high }}$ or $L I N-28 B^{\text {low }}$ let- $7 a^{\text {high }} I G F-I I{ }^{\text {high }}(p<0.0001$ for overall, $p=0.017$ for progression-free survival, respectively). Multivariate Cox regression models showed that

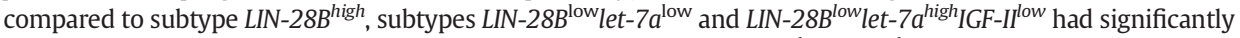
reduced mortality and reduced relapse risks. Moreover, subtype $L I N-28 B^{\text {low }}$ let- $7 a^{\text {low }}$ had better response to chemotherapy than subtype LIN-28B ${ }^{\text {high }}$.

Conclusions. These results suggest that molecular subtypes of the LIN-28B/let-7a/IGF-II axis associate with heterogeneous progression and may have clinical implications in predicting epithelial ovarian cancer prognosis. (c) 2016 Elsevier Inc. All rights reserved.
Abbreviations: CART, Classification and Regression Tree; cDNA, complementary DNA $\mathrm{CI}$, confidence interval; CSD, cold-shock domain; EI, expression index; EMT, epithelialmesenchymal transition; EOC, epithelial ovarian cancer; FIGO, International Federation of Gynecology and Obstetrics; HR, hazard ratio; IGF-II, insulin-like growth factor-II IGF2BPs, IGF-II mRNA binding proteins; LOI, loss of imprinting; ORs, odds ratios; PCR, polymerase chain reaction; Pri-let-7a, primary let-7a; WHO, World Health Organization; ZFMs, zinc finger domains.

* Corresponding author at: Department of Chronic Disease Epidemiology, School of Public Health, School of Medicine, Yale Cancer Center, Yale University, 60 College Street, New Haven, CT 06520-8034, USA.

E-mail address: lingeng.lu@yale.edu (L. Lu).

\section{Introduction}

LIN-28B, a paralog of LIN-28A, is a cancer stem cell-associated transcription factor, and plays an important role in maintaining stem celllike properties and tumorigenicity [1,2]. LIN-28B was first identified in human liver cancer [2], and shares $77 \%$ identity in protein sequence with LIN-28A. It contains a cold-shock domain (CSD) and two retroviral-type CCHC zinc finger domains (ZFMs) [3,4]. A negative feedback regulatory loop exists between LIN-28B and miRNA let-7a. With RNA-binding domains, LIN-28B is able to affect the biogenesis of miRNA let-7 members with a slightly stronger effect on let-7a, reducing let-7a maturation and its abundance by sequestering primary let-7a (pri-let-7a) in the nucleus [3,5], and thereby activating its many oncogene targets including k-RAS, c-MYC, HMGA2, cyclin D1, insulin-like 
growth factor-II (IGF-II), and IGF-II mRNA binding proteins (IGF2BPs) through the complementary binding sites of let-7a to the 3'-UTRs [5-7]. Conversely, miRNA let-7a can also, in turn, control the expression of $L I N-28 B$ [2], and indirectly affects the stability of LIN-28B protein via the ubiquitin ligase TRIM71 via let-7a binding sites in its 3'-UTR [8]. Inhibition of LIN-28B promotes the maturation of let-7, thereby leading to the translation reduction of let-7a targets such as $c-M Y C$ and $R A S$, and the decreased tumorigenesis [9]. In addition, the critical role of LIN28B/let-7 axis has been demonstrated in programing and maintaining stem cells [10,11], as well as in promoting epithelial-mesenchymal transition (EMT) [12], tumorigenesis and metastasis [11]. The involvement of the LIN-28B/let-7 axis in cancer has also been studied in vivo neuroblastoma animal model; $L I N-28 B$ overexpression-induced neuroblastma had markedly low levels of let-7 miRNA and high levels of MYC protein [13]. Frequent dysregulation of $L I N-28 B$ in human cancers including liver, breast, and ovary suggests that $L I N-28 B$ functions as an oncogene $[2,6,11,14,15]$, influencing disease prognosis and patient response to treatment [5].

Mitogenic growth factor IGF-II together with its receptor IGF-IR plays an important role in carcinogenesis and tumor progression. The association of IGF-II overexpression and increased risk of human cancer including ovarian cancer, and poor cancer survival has been reported previously [16,17]. Animal models and in vitro experiments show that IGF-IR inhibition can increase the efficacy of chemotherapy and suppress tumor-cell growth [18], suggesting that the suppression of IGFII/IGF-IR may benefit patients with malignancies. Besides loss of imprinting (LOI) and promoter methylation, LIN-28A/LIN-28B and miRNA let-7a also contribute to the regulation of IGF-II expression [7, $19,20]$. Elevated let-7a expression has been positively associated with increased expression of IGF-II [7,20]. In addition to its ability to suppress let-7a maturation, LIN-28B is also involved in regulating mRNA targets, such as IGF-II, HMGA1, OCT4, and cyclins via let-7a-independent mechanisms [21]. A positive correlation has been observed between the expressions of $L I N-28 B$ and IGF-II in cancer tissues [5]. It has been reported that the LIN-28B/IGF-II axis is linked to the progression of head and neck cancer [22]. Furthermore, Kallen and colleagues recently showed that miRNA let-7a activity can be antagonized by long noncoding RNA H19 [23], which is regulated with its neighboring IGF-II gene through a reciprocal DNA methylation-dependent mechanism in the imprinting control region. Through the direct or indirect regulation network, RNA protein LIN-28B, miRNA let-7a and the growth factor IGFII form an axis, which in different combinations may drive differences in phenotypes of human diseases including human cancer, given that each component of the axis has been shown in association with development, cell proliferation and apoptosis $[1,2,6,7,24]$. We asked whether ovarian cancer patients with heterogeneous phenotypes had different molecular subtypes of the axis. Thus, we evaluated the associations between the LIN-28B/let-7a/IGF-II axis molecular subtypes and both the clinicopathologic characteristics and survival of patients with epithelial ovarian cancer.

\section{Materials and methods}

\subsection{Patients and tumor samples}

With the approval of the ethics review committee and signed informed consent of patients, fresh tumor samples were collected from 211 epithelial ovarian cancer patients when they received cytoreduction surgery for primary ovarian cancer in the Department of Gynecology and Obstetrics at the University of Turin between October 1991 and February 2000. The specimens were snap-frozen in liquid nitrogen immediately after resection, and then transferred to a $-80^{\circ} \mathrm{C}$ freezer for storage. Clinical and pathology information on these patients was retrieved from their medical charts and pathology reports. Disease stage, tumor grade and histology were determined based on the International Federation of Gynecology and
Obstetrics (FIGO) classification and the WHO guidelines [25,26]. Most of the patients $(n=178)$ received standard post-operative platinum-based chemotherapy after cytoreduction surgery. For patients treated between 1991 and 1995, cisplatin $\left(75 \mathrm{mg} / \mathrm{m}^{2}\right)$ and cyclophosphamide $\left(750 \mathrm{mg} / \mathrm{m}^{2}\right)$ were administered every 3 weeks for 6 cycles as the standard of care except for patients who had allergic reactions or medical contraindications. From 1996, when paclitaxel was introduced as standard of care, patients were treated with paclitaxel ( $175 \mathrm{mg} / \mathrm{m}^{2}, 3 \mathrm{~h}$ infusion) and carboplatin at an area under the concentration time curve of 5 or 6 , every 3 weeks for 6 cycles. Patient response to chemotherapy was evaluated 1 month after the last cycle of treatment through clinical examination, imaging and serum CA-125. Pathologic evaluation by second-look laparotomy was performed for some patients following the institutional protocols at the time. Patients were subsequently followed through June 2001 for disease progression. The median follow-up time was 31 months with a range from 0.6 to 114 months.

"Measurable disease" was determined following the WHO criteria [27]. Four categories of patient response to chemotherapy were defined in this study, including (a) complete response: resolution of all evidence of the disease for at least one month, (b) partial response: a decrease of $\geq 50 \%$ in the product of the diameters (maximum and minimum) of all measurable lesions without the development of new lesions for at least one month, (c) stable disease: a decrease of $<50 \%$ or an increase of $<25 \%$ in the product of the diameters of all measurable lesion, and (d) progressive disease: an increase of $\geq 25 \%$ in the product of the diameters of all measurable lesions or the development of new lesions. For non-measurable disease, progression was defined as the doubling of CA-125 from the upper limit of normal [28]. Of 178 patients receiving chemotherapy, 176 had available information on chemotherapy response; 128 (72.7\%) who had complete response were classified as 'Yes' to treatment response, while 48 (27.3\%) who were in the other three categories were grouped as 'No' response, which included 36 with partial response, 4 with stable disease, and 8 with progressive disease.

\subsection{Total RNA extraction and analysis of lin-28B, let-7a and IGF-II expressions}

Frozen tumor specimens, which had been examined by two independent pathologists to confirm that each specimen contained greater than $80 \%$ tumor cells, were pulverized manually in liquid nitrogen, and approximately $100 \mathrm{mg}$ samples of tissue powder were used for extraction of total RNA through a standard phenol-chloroform approach. The quality of RNase-free DNase-treated total RNA was determined after $1 \%$ agarose gel electrophoresis for the integrity of $18 \mathrm{~s}$ and $28 \mathrm{~s}$ rRNA, and the quantity of the extracted RNA samples was determined by spectrophotometer.

Cloned AMV First-Strand cDNA synthesis kits (Invitrogen, Carlsbad, CA) were used to prepare cDNA. SYBR green-based real-time PCR was performed to determine the mRNA levels of LIN-28B and IGF-II. The qPCR was carried out as described elsewhere [5,16], including information on primer sequences. Briefly, in a volume of $20 \mu \mathrm{PCR}$ reaction, $1 \mu \mathrm{l}$ of cDNA template was mixed with $10 \mu \mathrm{l}$ of $2 \times$ Power SYBR ${ }^{\circ}$ PCR master mix (Applied Biosystems, Foster City, CA), $200 \mathrm{nM}$ of paired primers of either target genes of LIN-28B or IGF-II or internal controls of RNU48 and GAPDH, and distilled water. The PCR amplification included initial incubation at $50{ }^{\circ} \mathrm{C}$ for $2 \mathrm{~min}$, denaturing at $95^{\circ} \mathrm{C}$ for $10 \mathrm{~min}$, and 40 cycles of denaturing at $95^{\circ} \mathrm{C}$ for $15 \mathrm{~s}$ and annealing at $60^{\circ} \mathrm{C}$ for $1 \mathrm{~min}$. Melting curves were analyzed after each run to verify the size of the PCR products.

The miRNA cDNAs were prepared using TaqMan ${ }^{\circledR}$ microRNA Reverse Transcription kits (Applied Biosystems) as described elsewhere [7]. Analysis of let-7a expression in tumor tissue was performed using TaqMan ${ }^{\circledR}$ microRNA assay (Applied Biosystems) following the manufacturer's instructions. Briefly, levels of let-7a and RNU48 (an 
internal control for normalization) expression in the samples were determined with the TaqMan ${ }^{\circledR}$ miRNA assay (Applied Biosystems). In the PCR reaction $(15 \mu \mathrm{l}), 0.3 \mu \mathrm{l}$ of cDNA template was mixed with $7.5 \mu \mathrm{l}$ of $2 \mathrm{X}$ TaqMan ${ }^{\circledR}$ Universal PCR master mix (Applied Biosystems), $0.75 \mu \mathrm{l}$ of 20X probe/primers (Applied Biosystems) of either let-7a or RNU48, and water. The PCR amplification conditions were the same as the quantification of LIN-28B and IGF-II in this study.

All qPCR assays were completed using the Chromo4 ${ }^{\mathrm{TM}}$ Real-time PCR System (MJ Research Inc., Waltham, MA). Each sample was analyzed in duplicate, and the analysis was repeated for those with CV above $5 \%$.

\subsection{Statistical analysis}

Expressions of LIN-28B, let-7a and IGF-II were quantified by means of expression index (EI), which was calculated based on the formula $1000 \times 2^{(-\Delta \mathrm{Ct})}$, where $\Delta \mathrm{Ct}=\mathrm{Ct}_{\text {target gene }}-\mathrm{Ct}_{\text {internal control }}$. Classification and Regression Tree (CART) was constructed in each branch using the optimal cutoff values of LIN-28B, let-7a and IGF-II expressions, a point best distinguishing survivors and non-survivors, which was determined by an unsupervised algorithm of the maximization of hazard ratio as described elsewhere [29]. Associations between clinicopathologic features and the CART subtypes were analyzed using the Chi-square statistic or general linear model. Unconditional logistic regression analyses were performed to estimate odds ratios (ORs) and their corresponding 95\% confidence intervals (95\% CIs) for the associations of $L I N-28 B / l e t-7 a /$ IGF-II axis expression and clinicopathologic variables. Cox proportional hazards regression survival analyses were also conducted to assess the associations of the subtypes with risks of disease progression and mortality. The survival times for overall and disease progression-free survival were calculated as time from surgery until death or relapse, or the last follow-up, respectively. All statistical analyses were carried out using SAS version 9.3. A two-tailed $p$ value less than 0.05 was considered statistically significant, and the results were highlighted in bold in tables.

\section{Results}

\subsection{Clinical and pathologic characteristics of patients}

Table 1 shows the clinical and pathologic features of patients. Patient age at surgery was 57.9 years on average (range: $26-82$ ). The majority of patients had a disease with advanced stage and poor differentiation.

\subsection{LIN-28B, let-7a and IGF-II expressions and CART analysis in epithelial ovarian cancer}

Due to the limited tumor tissue samples, only 202 samples provided sufficient RNAs for IGF-II assay. The average EIs of LIN-28B, let-7a and IGF-II expression levels were 0.01 (5th - 95th percentile range: 0-6.0), 4.63 (range: $0.53-35.3$ ) and 11.07 (range: 0-3834), respectively (Table 1). In our previous studies, we reported high levels of $L I N-28 B$ and IGF-II expression associated with increased risk of relapse and overall mortality [5,16], and high let-7a was related to these increased risks in patients receiving platinum and paclitaxel treatment. However, an opposite effect of high let-7a on survival outcomes was observed in patients treated with platinum without paclitaxel [30]. To investigate the effects of gene-gene interaction on patient survival, we performed CART analysis. Fig. 1 shows the results of CART analysis focusing on LIN-28B/let-7a/IGF-II expressions. A total number of 197 patients had full data available for both gene expressions and vital status. Of 197 patients, 91 died of the disease, and 93 had recurrence. Using an unsupervised algorithm of the maximization of the hazard ratio for overall mortality, the optimal cutoff values for LIN-28B, let-7a and IGF-II were determined. The cutoff value for $L I N-28 B$ was 0.125 EI which classified patients into two groups, one (named terminal subtype \#1) containing 72 patients with $L I N-28 B \geq 0.125 \mathrm{EI}($ LIN-28B high $)$, and the other 125 patients with $L I N-28 B<0.125 \mathrm{EI}\left(L I N-28 B^{\text {low }}\right)$. Further classification of the
Table 1

Clinical and pathologic characteristics of patients.

\begin{tabular}{|c|c|c|}
\hline Variables & $\mathrm{N}$ & $\%$ \\
\hline Age (years), Mean (range) & 208 & $57.9(26-82)$ \\
\hline Tumor Grade & 211 & \\
\hline 1 & 34 & 16.1 \\
\hline 2 & 40 & 19.0 \\
\hline 3 & 137 & 64.9 \\
\hline Disease stage & 211 & \\
\hline I & 52 & 24.6 \\
\hline II & 12 & 5.7 \\
\hline III & 133 & 63.0 \\
\hline IV & 14 & 6.6 \\
\hline Residual tumor size (cm) & 207 & \\
\hline 0 & 91 & 44.0 \\
\hline$>0$ & 116 & 56.0 \\
\hline Histological type & 211 & \\
\hline Serous Papillary & 85 & 40.3 \\
\hline Endometrioid & 41 & 19.4 \\
\hline Undifferentiated & 36 & 17.1 \\
\hline Mucinous & 18 & 8.5 \\
\hline Clear Cell & 16 & 7.6 \\
\hline Müllerian & 14 & 6.6 \\
\hline Other & 1 & 0.5 \\
\hline Debulking results & 208 & \\
\hline Optimal & 108 & 51.9 \\
\hline Suboptimal & 100 & 48.1 \\
\hline Chemotherapy response & 176 & \\
\hline No & 48 & 27.3 \\
\hline Yes & 128 & 72.7 \\
\hline Gene expression & $\mathrm{N}$ & Median (5th-95th range) \\
\hline$L I N-28 B$ & 211 & $0.01(0-6.00)$ \\
\hline Let-7a & 211 & $4.63(0.53-35.3)$ \\
\hline IGF-II & 202 & $11.07(0-3834)$ \\
\hline
\end{tabular}

latter group with a cutoff value of let-7a at $1.32 \mathrm{EI}, 22$ of the 125 patients with let-7a $<1.32 \mathrm{EI}\left(\right.$ let-7 $\left.a^{\text {low }}\right)$ were grouped into terminal subtype \#2 $\left(\right.$ LIN-28B ${ }^{\text {low }}$ let- $\left.7 a^{\text {low }}\right)$, and 103 patients with let-7a $\geq 1.32 \mathrm{EI}\left(\right.$ let-7a $\left.{ }^{\text {high }}\right)$ in another group which was further divided by IGF-II levels. The IGF-II cutoff value was $6.85 \mathrm{EI}$, which classified the patients with low LIN$28 \mathrm{~B}(<0.125 \mathrm{EI})$ but high let-7a $(\geq 1.32 \mathrm{EI})$ into two subtypes, subtype \#3 (LIN-28B ${ }^{\text {low }}$ let-7a $a^{\text {high }}$ IGF-II ${ }^{\text {low }}$ ) having 56 patients with IGF-II $<6.85$ EI (IGF-II $\left.{ }^{\text {low }}\right)$, and subtype \#4 (LIN-28B low let- $7^{\text {high }}$ IGF-II $\left.{ }^{\text {high }}\right)$, with 47 patients with $I G F-I I \geq 6.85 \mathrm{EI}\left(I G F-I I^{\text {high }}\right)$. No further classification of the subtype \#1 was based on the evidence that high LIN-28B, a cancer stem cell-associated gene, was associated with poor prognosis of human cancer such as ovary and liver $[1,5,6]$. In addition, no significant differences in disease survival were observed across the subgroups when we used the same approach to classifying the subtype \#1 into different subgroups (data not shown).

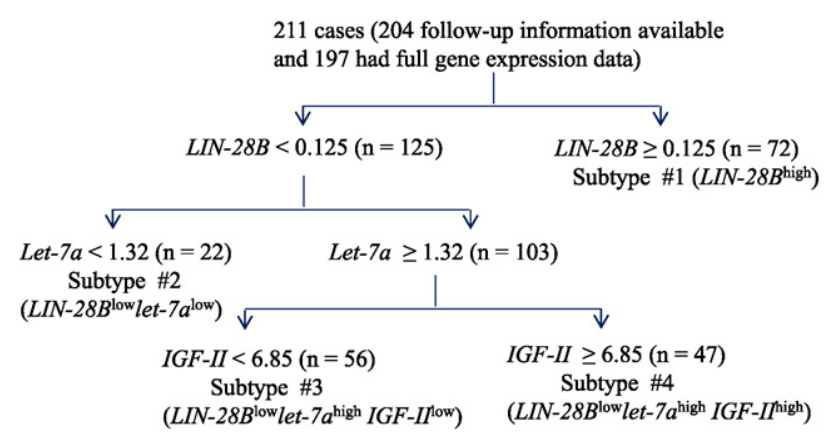

Fig. 1. Classification and Regression Tree (CART) analysis of the LIN-28B/let-7a/IGF-II axis. The cutoff values for LIN-28B, let-7a and IGF-II expression levels were determined by an unsupervised algorithm for the maximization of the hazard ratio differentiating survivors from non-survivors as described in the Materials and Methods. The terminal branches were named as subtypes. ' $n$ ' is the number of patients in a particular branch. 
3.3. Associations of LIN-28B/let-7a/IGF-II axis molecular subtypes with clincopathologic features of epithelial ovarian cancer

We first evaluated the associations of the LIN-28B/let-7a/IGF-II axis molecular subtypes with clinicopathologic features of 197 patients with both gene expressions and vital status; the results are shown in Table 2. Borderline significant difference in patient age was found across the subtypes ( $p=0.050$ ), and patients with the subtype \#3 showed younger. We found significant associations of the axis with disease stage ( $p=0.037)$, residual tumor size $(p=0.001)$ and debulking results $(p=0.001)$, and no significant associations with tumor grade ( $p=0.112)$, chemotherapy response $(p=0.064)$, and histological type $(p=0.074)$. To assess the strength of associations between the CART subtypes and clinicopathologic features, we performed unconditional logistical regression analyses with the adjustment for patient age at surgery. Across all the features analyzed, no statistically significant differences were found between subtype \#4 and \#1. However, patients in subtypes \#2 and \#3 were less likely to have larger residual tumors or suboptimal debulking results compared to those in subtype $\# 1$. The adjusted odds ratios (ORs) for subtype \#2 patients were 0.17 (95\% CI: 0.06-0.49) and 0.15 (95\% CI: 0.05-0.49), and for subtype \#3 patients were 0.35 (95\% CI: 0.17-0.75) and 0.44 (95\% CI: 0.21-0.90) with regard to having larger residual tumors or suboptimal debulking results, respectively. We also found that patients in subtype \#2 were less likely to have advanced disease stage or high tumor grade and more like to respond to chemotherapy compared to those in subtype \#1; their adjusted ORs were 0.27 (95\% CI: 0.10-0.73), 0.34 (95\% CI: 0.13-0.92), and 8.77 (95\% CI: 1.09-70.4), respectively. In addition, patients in subtype \#3 were less likely to have serous tumors or type II (based on the classification defined by Kurman and colleagues [31]) compared to those in subtype \#1. The adjusted ORs were 0.38 (95\% CI: 0.18-0.81), and 0.26 (95\% CI: 0.11-0.65), respectively.

\subsection{Associations of LIN-28B/let-7a/IGF-II axis molecular subtypes with patient survival}

Kaplan-Meier survival curves according to the LIN-28B/let-7a/IGF-II axis subtypes were constructed for overall and for disease progression free survival. Figs. 2 and 3 show that both subtypes \#2 and \#3 had significantly better overall and disease progression free survival than subtypes \#1 and \#4 ( $p<0.0001$ and $p=0.017$, respectively). The overall survival curves did not differ significantly between subtypes \#2 and \#3; neither did those differ between subtypes \#1 and \#4. The disease progression free survival curves were also not separable between subtypes \#2 and \#3 until the end of 60-month follow-up, and for subtypes \#1 and \#4 the curves were not distinct throughout the follow-up. The associations remained significant when only the patients receiving chemotherapy were included in Kaplan-Meier survival curve analyses ( $p=0.0001$ for overall survival, and $p=0.040$ for disease progression free survival) (supplementary Figs. 1 and 2).

To confirm the results of Kaplan-Meier survival analysis and to adjust for potential confounding factors, we further analyzed the data with multivariate Cox proportional hazards regression models. The results of the multivariate analyses are shown in Table 3. Compared to subtype \#1, subtypes \#2 $(p=0.038)$ and \#3 $(p<0.0001)$ but not subtype \#4 ( $\mathrm{p}=0.144)$ had significantly decreased mortality risks with adjustment for patient age at surgery, disease stage, tumor grade, residual tumor size, histological type and chemotherapy

Table 2

Associations of the LIN-28B/let-7a/IGF-II axis molecular subtypes with clinicopathologic features.

\begin{tabular}{|c|c|c|c|c|c|c|}
\hline Variable & $\mathrm{N}$ & $\begin{array}{l}\text { Subtype }{ }^{1} \# 1 \\
\text { n (\%) }\end{array}$ & $\begin{array}{l}\text { Subtype \#2 } \\
\text { n (\%) }\end{array}$ & $\begin{array}{l}\text { Subtype \#3 } \\
\text { n (\%) }\end{array}$ & $\begin{array}{l}\text { Subtype \#4 } \\
\text { n (\%) }\end{array}$ & $p$ value \\
\hline $\mathrm{Age}^{2}($ mean $\pm S D$, years) $(\mathrm{n})$ & 197 & $59.2 \pm 11.3(72)$ & $61.5 \pm 8.5(22)$ & $54.8 \pm 11.2(56)$ & $58.8 \pm 11.5(47)$ & 0.050 \\
\hline Disease stage & 197 & & & & & 0.037 \\
\hline I-II & 56 & $15(26.8)$ & $11(19.6)$ & $19(33.9)$ & $11(19.6)$ & \\
\hline III-IV & 141 & $57(40.4)$ & $11(7.8)$ & $37(26.2)$ & $36(25.5)$ & \\
\hline $\mathrm{OR}^{3}\left(95 \% \mathrm{Cl}^{4}\right)$ & & Reference & $0.27(0.10-0.73)$ & $0.50(0.23-1.13)$ & $0.86(0.36-2.08)$ & \\
\hline Tumor grade & 197 & & & & & 0.112 \\
\hline 1 & 67 & $19(28.4)$ & $11(16.4)$ & $23(34.3)$ & $14(20.9)$ & \\
\hline $2-3$ & 130 & $53(40.8)$ & $11(8.5)$ & $33(25.4)$ & $33(25.4)$ & \\
\hline OR $(95 \% \mathrm{CI})$ & & Reference & $0.34(0.13-0.92)$ & $0.55(0.26-1.18)$ & $0.85(0.38-21.93)$ & \\
\hline Residual tumor size $(\mathrm{cm})$ & 197 & & & & & 0.001 \\
\hline 0 & 83 & $22(26.5)$ & $16(19.3)$ & $30(36.1)$ & $15(18.1)$ & \\
\hline$>0$ & 114 & $50(43.9)$ & $6(5.3)$ & $26(22.8)$ & $32(28.1)$ & \\
\hline OR $(95 \% \mathrm{CI})$ & & Reference & $0.17(0.06-0.49)$ & $0.35(0.17-0.75)$ & $0.93(0.42-2.06)$ & \\
\hline Histological type & 197 & & & & & 0.074 \\
\hline Non-serous & 117 & $35(29.9)$ & $13(11.1)$ & $40(34.2)$ & $29(24.8)$ & \\
\hline Serous & 80 & $37(46.3)$ & $9(11.3)$ & $16(20.0)$ & $18(22.5)$ & \\
\hline OR $(95 \% \mathrm{CI})$ & & Reference & $0.65(0.25-1.74)$ & $0.38(0.18-0.81)$ & $0.59(0.28-1.24)$ & \\
\hline Histological type $e^{5}$ & 197 & & & & & 0.014 \\
\hline Type I & 48 & $9(18.8)$ & $7(14.6)$ & $20(41.7)$ & $12(25.0)$ & \\
\hline Type II & 149 & $63(42.3)$ & $16(10.7)$ & $36(24.2)$ & $34(22.8)$ & \\
\hline OR $(95 \% \mathrm{CI})$ & & Reference & $0.33(0.10-1.00)$ & $0.26(0.11-0.65)$ & $0.40(0.16-1.06)$ & \\
\hline Debulking results & 197 & & & & & 0.001 \\
\hline Optimal & 99 & $29(29.3)$ & $18(18.2)$ & $34(34.3)$ & $18(18.2)$ & \\
\hline Suboptimal & 98 & $43(43.9)$ & $4(4.1)$ & $22(22.5)$ & $29(29.6)$ & \\
\hline OR $(95 \% \mathrm{CI})$ & & Reference & $0.15(0.05-0.49)$ & $0.44(0.21-0.90)$ & $1.09(0.51-2.31)$ & \\
\hline Chemotherapy response & 173 & & & & & 0.064 \\
\hline No & 47 & $21(44.7)$ & $1(2.1)$ & $12(25.5)$ & $13(27.7)$ & \\
\hline Yes & 126 & $42(33.3)$ & $19(14.3)$ & $36(28.6)$ & $29(23.8)$ & \\
\hline OR $(95 \% \mathrm{CI})$ & & Reference & $8.77(1.09-70.4)$ & $1.63(0.70-3.83)$ & $1.17(0.51-2.72)$ & \\
\hline
\end{tabular}

Note: The results with $p<0.05$ are highlighted in bold.

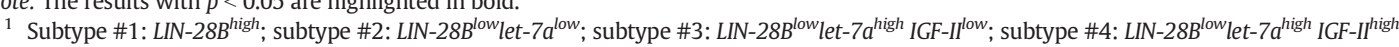

2 SD: standard deviation.

3 OR: odds ratio obtained from the unconditional logistical regression model adjusted with patient age at surgery, in which the second level of the variable (e.g., III-IV in the variable of disease stage) as the event in the dependent variable.

$4 \mathrm{Cl}$ : confidence interval.

5 Classification of type I and II tumors [31]: type I includes low-grade (grade 1) serous, low-grade endometrioid, clear cell, mucinous and Brenner; and type II includes high-grade (grade

2 and 3) serous, high-grade endometrioid, undifferentiated carcinoma and malignant mixed mesodermal (or Müllerian) tumors. 


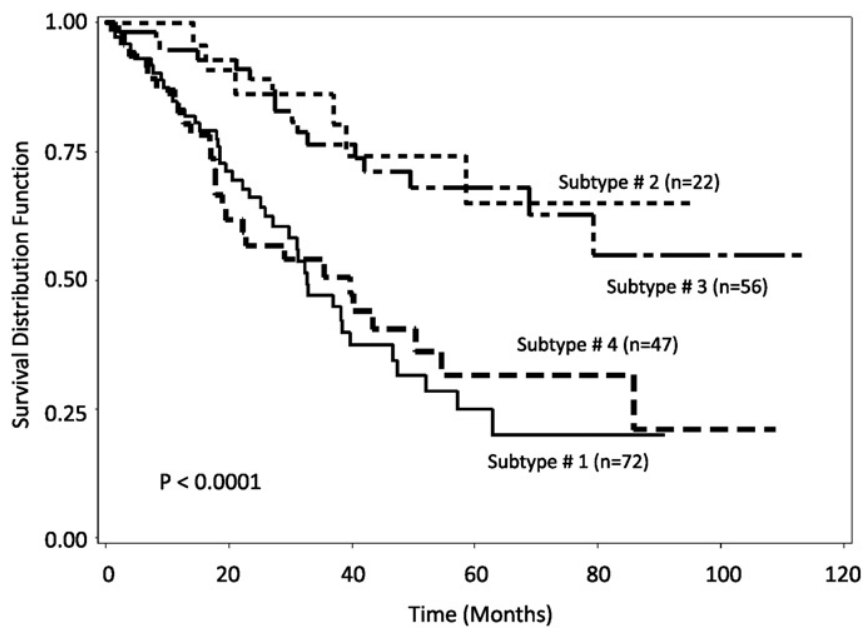

Fig. 2. Kaplan-Meier overall survival curves of epithelial ovarian cancer stratified by the molecular subtypes of the LIN-28B/let-7a/IGF-II axis. The curves for the subtypes \#2

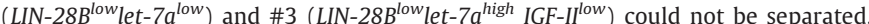
neither could curves for the subtypes \#1 $\left(L I N-28 B^{\text {high }}\right)$ and \#4 (LIN-28B $B^{\text {low }}$ let-7a ${ }^{\text {high }}$ IGF$\left.I I^{\text {high }}\right)$. Patients in subtypes \#2 and \#3 had better overall survival than those in the subtypes \#1 and \#4 ( $p<0.0001$, log-rank test).

status. The adjusted hazard ratios (HRs) of death were 0.36 (95\% CI: 0.14-0.94) for subtype \#2, 0.31 (95\% CI: 0.17-0.56) for subtype \#3, and 0.68 (95\% CI: 0.41-1.14) for subtype \#4. Compared to subtype \#1, subtypes \#2 and \#3 but not \#4 had significantly reduced risks of relapse. The adjusted HRs for relapse were 0.44 (95\% CI: 0.19-0.99) for subtype \#2 ( $p=0.047), 0.56$ (95\% CI: 0.33-0.96) for subtype \#3 $(p=0.035)$, and $0.86(95 \% \mathrm{CI}: 0.51-1.45)$ for subtype \#4 ( $p=0.563)$.

\section{Discussion}

In this study, we demonstrated the associations of the LIN-28B/let$7 a / I G F-I I$ axis molecular subtype with patient survival in epithelial ovarian cancer. Patients with either $L I N-28 B^{\text {high }}$ or $L I N-28 B^{\text {low }}$ let- $7 a^{\text {high }}$ IGF-II ${ }^{\text {high }}$ had aggressive disease and unfavorable survival compared to patients with either LIN-28B low let-7a low or LIN-28B ${ }^{\text {low }}$ let-7 $7 a^{\text {high }}$ IGF$I^{\text {low }}$. Moreover, patients in subtype \#2 who had the subtype of LIN-28B

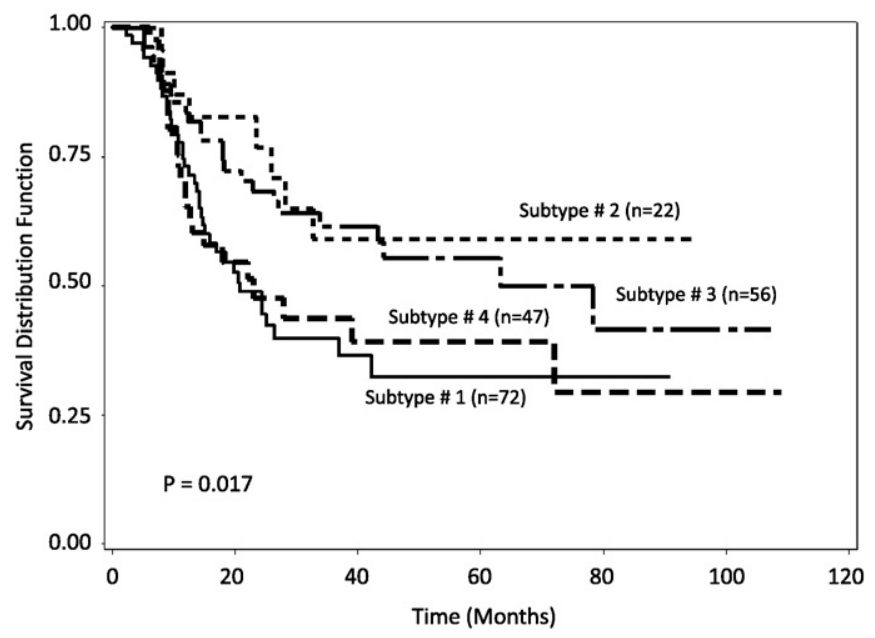

Fig. 3. Kaplan-Meier disease progression free survival curves of epithelial ovarian cancer stratified by the molecular subtypes of the LIN-28B/let-7a/IGF-II axis. The curves for the

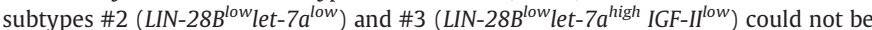
separated, neither could curves for the subtypes \#1 (LIN-28B high $)$ and \#4 (LIN-28B ${ }^{\text {low }}$ let$7 a^{\text {high }}$ IGF-II ${ }^{\text {high }}$ ). Patients in subtypes \#2 and \#3 had better disease progress free survival than those in the subtypes \#1 and \#4 ( $p=0.028$, log-rank test).
Table 3

Associations of LIN-28B/let-7a/IGF-II axis molecular subtypes and patient survival of epithelial ovarian cancer.

\begin{tabular}{|c|c|c|c|c|c|c|}
\hline \multirow[t]{2}{*}{ Variable } & \multicolumn{3}{|l|}{ Death } & \multicolumn{3}{|c|}{ Relapse } \\
\hline & $\mathrm{HR}^{1}$ & $95 \% \mathrm{CI}^{2}$ & $p$ value & HR & $95 \% \mathrm{CI}$ & $\begin{array}{l}p \\
\text { value }\end{array}$ \\
\hline \multicolumn{7}{|l|}{ Group $^{3}$} \\
\hline Subtype \#1 & 1.00 & & & 1.00 & & \\
\hline Subtype \#2 & 0.36 & $0.14-0.94$ & 0.038 & 0.44 & $0.19-0.99$ & 0.047 \\
\hline Subtype \#3 & 0.31 & $0.17-0.56$ & $<0.0001$ & 0.56 & $0.33-0.96$ & 0.035 \\
\hline Subtype \#4 & 0.68 & $0.41-1.14$ & 0.144 & 0.86 & $0.51-1.45$ & 0.563 \\
\hline Age & 1.00 & $0.98-1.02$ & 0.847 & 1.01 & $0.99-1.03$ & 0.484 \\
\hline Disease stage & 1.53 & $1.05-2.21$ & 0.026 & 1.44 & $1.03-2.02$ & 0.032 \\
\hline Tumor grade & 1.55 & $1.00-2.41$ & 0.050 & 1.27 & $0.87-1.84$ & 0.209 \\
\hline Residual tumor size & 1.26 & $1.16-1.37$ & $<0.0001$ & 1.11 & $1.02-1.20$ & 0.012 \\
\hline $\begin{array}{l}\text { Histological type } \\
\text { (Serous vs non-serous) }\end{array}$ & 0.801 & $0.52-1.27$ & 0.362 & 1.37 & $0.88-2.15$ & 0.166 \\
\hline $\begin{array}{l}\text { Chemotherapy (Yes vs } \\
\text { No) }\end{array}$ & 0.45 & $0.19-1.04$ & 0.061 & 1.69 & $0.51-5.57$ & 0.388 \\
\hline
\end{tabular}

Note: The results with $p<0.05$ are highlighted in bold.

1 HR: hazard ratio obtained from a multivariate Cox proportional hazard regression analysis, in which patient age, disease stage, tumor grade and residual tumor size are treated as numerical variables, while the group of the subtypes (\#2, \#3, \#4 vs \#1, respectively) obtained from the CART analysis, histological type (serous vs. non-serous) and chemotherapy status (yes vs no) are treated as categorical variables.

$2 \mathrm{CI}$ : confidence interval.

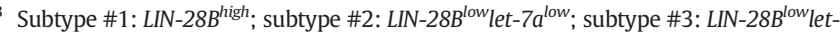
$7 a^{\text {high }}$ IGF-II ${ }^{\text {low }}$; subtype \#4: LIN-28B ${ }^{\text {low }}$ let-7 $a^{\text {high }}$ IGF-II ${ }^{\text {high }}$.

low $l e t-7 a^{\text {low }}$ had better response to chemotherapy that are effective in eliminating rapidly proliferating cancer cells. As shown in the results, patients in the subtype \#1 (LIN-28B $\left.{ }^{\text {high }}\right)$ had higher cancer stem cellassociated gene LIN-28B expression, and in the subtype \#4 (LIN-28B low let-7 ${ }^{\text {high }}$ IGF-II ${ }^{\text {high }}$ ) had particularly higher expression of IGF-II, a stem cell and cancer development-associated signaling, in comparison with the subtypes \#2 and \#3. These results suggest that different molecular mechanisms are underlying the progression of epithelial ovarian cancer in term of LIN-28B/let-7a/IGF-II axis.

LIN-28B has been suggested to be an oncogene [2,11], and its encoded protein is a transcription factor involved in maintaining properties of cancer stem cells [32], the culprit of the aggressiveness and metastasis of disease, and resistance to therapy. Hosonuma and colleagues [33] showed that ovarian cancer patients with stem cell-like side populations of tumor cells had worse prognoses than those without. In vitro and in vivo experiments show that progenitor-like cells make the behavior of human epithelial ovarian cancer aggressive [34]. Similarly, King and colleagues [35] showed that the activation of LIN-28B stimulated the expression of the intestinal/colonic epithelial stem cell markers LGR5 and PROM1, and promoted the migration, invasion and transformation of immortalized colonic epithelial cells. Another previous study also showed that down-regulation of $L I N-28 B$ could repress the self-renewal capability of prostate cancer cell lines [32]. Animal models indicate that LIN-28B activation promotes cancer growth; LIN-28B silencing significantly inhibits MYC-dependent cell proliferation [36]. In agreement with our present results, the association of high $L I N-28 B$ expression with poor clinical outcome has also been observed in other human cancers, including esophagus, and head and neck [22,37]. Moreover, in line with our findings that subtype \#1 with high LIN-28B expression had unfavorable clinical features of epithelial ovarian cancer, it has been reported that aberrant up-regulated $L I N-28 \mathrm{~B}$ expression is observed in subsets of tumors that are poorly differentiated [6,35].

Interestingly, we also found that subtype \#4, one of the branches with the subtype of $L I N-28 B^{\text {low }}$ let-7a $a^{\text {high }}$ IGF-II high , had worse survival than subtype \#3 of the LIN-28B low let-7a high IGF-II low. Moreover, although patients with low $L I N-28 B$ expression may have favorable prognosis compared to those with the high one, if patients also had high let-7a particularly high IGF-II expression, they also showed an inferior prognosis. This result indicates that IGF-II is an unfavorable marker in ovarian cancer prognosis as we previously reported [16], and also is 
consistent with other studies. Huang et al. [38] showed ovarian cancer patients with high IGF-II had shorter survival and unfavorable disease, as well as paclitaxel resistance. This group recently reported that IGF-II silencing could make drug resistant-ovarian cancer responsive to paclitaxel [39]. Slipicevic and colleagues analyzed IGF-II levels in post-chemotherapy ovarian cancer effusions, and found a negative association between IGF-II levels and patient survival [40]. An in vitro study revealed that human monoclonal antibodies against IGF-IR could suppress IGF-II-dependent ovarian cancer growth, and increase sensitivity to cisplatin in ovarian cancer xenograft models [18].

MicroRNA let-7a is one member of the let-7 family, which is wellcharacterized in fine-tuning gene expression, and plays important roles in cell proliferation, differentiation, apoptosis and metabolism [24]. Aberrant expression of let-7a has been reported in various types of human cancer [41], and is associated with cell proliferation, chemotherapy response, and patient survival $[30,42]$. Evidence suggests that let-7a may operate in favor of tumor progression in human tumors under certain conditions $[7,30]$. In the present study, we found that subtype \#3 (LIN-28B ${ }^{\text {low }}$ let-7 $7 a^{\text {high }}$ IGF-II ${ }^{\text {low }}$ ), despite high let-7a, had relatively better survival compared to subtype \#4 (LIN-28B ${ }^{\text {low }}$ let- $7 a^{\text {high }}$ IGF$\left.I I^{\text {high }}\right)$, which was also from the branch of high let-7a expression. This finding suggests that let-7a may play different roles in the prognosis of these two groups with different levels of IGF-II expression. In addition, it was recently reported that down-regulation of let-7a in the LIN-28B/ let-7a signaling could sensitize ovarian cancer response to chemotherapy [43]. This observation is in agreement with our finding of chemotherapy response in subtype \#2 patients. Taken together, the findings in our study suggest that LIN-28B, let-7a and IGF-II interplay with each other, forming a complicated axis. Thus, it may not be unexpected that patients with the molecular subtypes in different combinations of the axis had their distinct outcomes.

In summary, we have shown that patients with the signatures of either LIN-28B ${ }^{\text {high }}$ or $L I N-28 B^{\text {low }}$ let-7 $a^{\text {high }}$ IGF-II ${ }^{\text {high }}$ had worse prognoses and unfavorable disease in comparison to those with either the

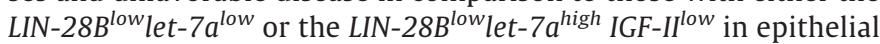
ovarian cancer. The results show that different molecular subtypes of the $L I N-28 B /$ let-7a/IGF-II axis have distinct progression courses, suggesting that the classification of the $L I N-28 B /$ let-7a/IGF-II axis subtypes may help to select patients in clinical trials to explore systemic or targeted therapies that can prevent or delay relapse and improve survival, and that the axis may have potential in designing precision medications for epithelial ovarian cancer. For example, patients with the signature $L I N-28 B^{\text {low }} l e t-7 a^{\text {high }} I G F-I I^{\text {high }}$ should be treated as aggressively as those with LIN-28B high , but different targets should be relevant for these two subgroups in designing target therapies. Similarly, individualized target therapy strategies may be necessary for patients with $L I N-28 B^{\text {low }}$ let $-7 a^{\text {low }}$ or LIN-28B ${ }^{\text {low }}$ let$7 a^{\text {high }}$ IGF-II ${ }^{\text {low }}$, although their prognoses are similar.

\section{Conflict of Interest Statement}

The authors declare that they have no conflicts of interest.

\section{Appendix A. Supplementary data}

Supplementary data to this article can be found online at http://dx. doi.org/10.1016/j.ygyno.2015.12.035.

\section{References}

[1] L. Lu, D. Katsaros, S.T. Mayne, H.A. Risch C. Benedetto, E.M. Canuto, et al., Functional study of risk loci of stem cell-associated gene lin-28B and associations with disease survival outcomes in epithelial ovarian cancer, Carcinogenesis 33 (2012) 2119-2125.

[2] Y. Guo, Y. Chen, H. Ito, A. Watanabe, X. Ge, T. Kodama, et al., Identification and characterization of lin-28 homolog B (LIN28B) in human hepatocellular carcinoma, Gene 384 (2006) 51-61.

[3] S.R. Viswanathan, G.O. Daley, R.I. Gregory, Selective blockade of microRNA processing by Lin28, Science 320 (2008) 97-100.
[4] L. Zhang, S. Volinia, T. Bonome, G.A. Calin, J. Greshock, N. Yang, et al., Genomic and epigenetic alterations deregulate microRNA expression in human epithelial ovarian cancer, Proc. Natl. Acad. Sci. U. S. A. 105 (2008) 7004-7009.

[5] L. Lu, D. Katsaros, K. Shaverdashvili, B. Qian, Y. Wu, I.A. de la Longrais, et al., Pluripotent factor lin-28 and its homologue lin-28b in epithelial ovarian cancer and their associations with disease outcomes and expression of let-7a and IGF-II, Eur. J. Cancer 45 (2009) 2212-2218.

[6] S.R. Viswanathan, J.T. Powers, W. Einhorn, Y. Hoshida, T.L. Ng, S. Toffanin, et al., Lin28 promotes transformation and is associated with advanced human malignancies, Nat. Genet. 41 (2009) 843-848.

[7] L. Lu, D. Katsaros, I.A. de la Longrais, O. Sochirca, H. Yu, Hypermethylation of let-7a-3 in epithelial ovarian cancer is associated with low insulin-like growth factor-II expression and favorable prognosis, Cancer Res. 67 (2007) 10117-10122.

[8] S.H. Lee, S. Cho, M. Sun Kim, K. Choi, J.Y. Cho, H.S. Gwak, et al., The ubiquitin ligase human TRIM71 regulates let-7 microRNA biogenesis via modulation of Lin28B protein, Biochim. Biophys. Acta 2014 (1839) 374-386.

[9] S. Segalla, S. Pivetti, K. Todoerti, M.A. Chudzik, E.C. Giuliani, F. Lazzaro, et al., The ribonuclease DIS3 promotes let-7 miRNA maturation by degrading the pluripotency factor LIN28B mRNA, Nucleic Acids Res. (2015).

[10] M.R. Copley, S. Babovic, C. Benz, D.J. Knapp, P.A. Beer, D.G. Kent, et al., The Lin28b-let-7-Hmga2 axis determines the higher self-renewal potential of fetal haematopoietic stem cells, Nat. Cell Biol. 15 (2013) 916-925.

[11] B.B. Madison, Q. Liu, X. Zhong, C.M. Hahn, N. Lin, M.J. Emmett, et al., LIN28B promotes growth and tumorigenesis of the intestinal epithelium via Let-7, Genes Dev. 27 (2013) 2233-2245

[12] L. Guo, C. Chen, M. Shi, F. Wang, X. Chen, D. Diao, et al., Stat3-coordinated Lin-28-let7-HMGA2 and miR-200-ZEB1 circuits initiate and maintain oncostatin M-driven epithelial-mesenchymal transition, Oncogene 32 (2013) 5272-5282.

[13] J.J. Molenaar, R. Domingo-Fernandez, M.E. Ebus, S. Lindner, J. Koster, K. Drabek, et al. LIN28B induces neuroblastoma and enhances MYCN levels via let-7 suppression, Nat. Genet. 44 (2012) 1199-1206.

14] E. Piskounova, C. Polytarchou, J.E. Thornton, R.J. LaPierre, C. Pothoulakis, J.P. Hagan, et al., Lin28A and Lin28B inhibit let-7 microRNA biogenesis by distinct mechanisms, Cell 147 (2011) 1066-1079.

[15] A. Helland, M.S. Anglesio, J. George, P.A. Cowin, C.N. Johnstone, House CM, et al., Deregulation of MYCN, LIN28B and LET7 in a molecular subtype of aggressive highgrade serous ovarian cancers, PLoS One 6 (2011), e18064.

[16] L. Lu, D. Katsaros, A. Wiley, I.A. de la Longrais, H.A. Risch, M. Puopolo, The relationship of insulin-like growth factor-II, insulin-like growth factor binding protein-3, and estrogen receptor-alpha expression to disease progression in epithelial ovarian cancer, Clin. Cancer Res. 12 (2006) 1208-1214.

[17] Y.F. Huang, W.F. Cheng, Y.P. Wu, Y.M. Cheng, K.F. Hsu, C.Y. Chou, Circulating IGF system and treatment outcome in epithelial ovarian cancer, Endocr. Relat. Cancer 21 (2014) 217-229.

[18] P.J. Beltran, F.J. Calzone, P. Mitchell, Y.A. Chung, E. Cajulis, G. Moody, et al. Ganitumab (AMG 479) inhibits IGF-II-dependent ovarian cancer growth and potentiates platinum-based chemotherapy, Clin. Cancer Res. 20 (2014) 2947-2958.

[19] P. Jelinic, P. Shaw, Loss of imprinting and cancer, J. Pathol. 211 (2007) 261-268.

[20] L. Lu, D. Katsaros, Y. Zhu, A. Hoffman, S. Luca, C.E. Marion, et al., Let-7a regulation of insulin-like growth factors in breast cancer, Breast Cancer Res. Treat. 126 (2011) 687-694

[21] A. Polesskaya, S. Cuvellier, I. Naguibneva, A. Duquet, E.G. Moss, A. Harel-Bellan, Lin28 binds IGF-2 mRNA and participates in skeletal myogenesis by increasing translation efficiency, Genes Dev. 21 (2007) 1125-1138.

[22] N.M. Alajez, W. Shi, D. Wong, M. Lenarduzzi, J. Waldron, I. Weinreb, et al., Lin28b promotes head and neck cancer progression via modulation of the insulin-like growth factor survival pathway, Oncotarget 3 (2012) 1641-1652.

[23] A.N. Kallen, X.B. Zhou, J. Xu, C. Qiao, J. Ma, L. Yan, et al., The imprinted H19 lncRNA antagonizes let-7 microRNAs, Mol. Cell 52 (2013) 101-112.

[24] H. Zhu, N. Shyh-Chang, A.V. Segre, G. Shinoda, S.P. Shah, W.S. Einhorn, et al., The Lin28/let-7 axis regulates glucose metabolism, Cell 147 (2011) 81-94.

[25] R. Scully, L. Sobin, S. Serov, Histological Typing of Ovarian Tumors, second ed Springer, Berlin (NY), 1999.

[26] J.H. Shepherd, Revised FIGO staging for gynaecological cancer, Br. J. Obstet Gynaecol. 96 (1989) 889-892.

[27] A.B. Miller, B. Hoogstraten, M. Staquet, A. Winkler, Reporting results of cancer treatment, Cancer 47 (1981) 207-214.

[28] G.J. Rustin, A.E. Nelstrop, M.K. Tuxen, H.E. Lambert, Defining progression of ovarian carcinoma during follow-up according to CA 125: a North Thames Ovary Group Study, Ann. Oncol. 7 (1996) 361-364.

[29] C.O.Q.J. Contal, An application of changepoint methods in studying the effect of age on survival in breast cancer, Comput. Stat. Data Anal. 30 (1999) 253-270.

[30] L. Lu, P. Schwartz, L. Scarampi, T. Rutherford, E.M. Canuto, H. Yu, et al., MicroRNA let7a: a potential marker for selection of paclitaxel in ovarian cancer management, Gynecol. Oncol. 122 (2011) 366-371.

[31] R.J. Kurman, I.M. Shih, The origin and pathogenesis of epithelial ovarian cancer: a proposed unifying theory, Am. J. Surg. Pathol. 34 (2010) 433-443.

[32] D. Kong, S. Banerjee, A. Ahmad, Y. Li, Z. Wang, S. Sethi, et al., Epithelial to mesenchymal transition is mechanistically linked with stem cell signatures in prostate cancer cells, PLoS One 5 (2010), e12445.

[33] S. Hosonuma, Y. Kobayashi, S. Kojo, H. Wada, K. Seino, K. Kiguchi, et al., Clinical significance of side population in ovarian cancer cells, Hum. Cell 24 (2011) 9-12.

[34] S.A. Bapat, A.M. Mali, C.B. Koppikar, N.K. Kurrey, Stem and progenitor-like cells contribute to the aggressive behavior of human epithelial ovarian cancer, Cancer Res. 65 (2005) 3025-3029. 
[35] C.E. King, M. Cuatrecasas, A. Castells, A.R. Sepulveda, J.S. Lee, A.K. Rustgi, LIN28B promotes colon cancer progression and metastasis, Cancer Res. 71 (2011) 4260-4268.

[36] T.C. Chang, L.R. Zeitels, H.W. Hwang, R.R. Chivukula, E.A. Wentzel, M. Dews, et al., Lin-28B transactivation is necessary for Myc-mediated let-7 repression and proliferation, Proc. Natl. Acad. Sci. U. S. A. 106 (2009) 3384-3389.

[37] R. Hamano, H. Miyata, M. Yamasaki, K. Sugimura, K. Tanaka, Y. Kurokawa, et al., High expression of Lin28 is associated with tumour aggressiveness and poor prognosis of patients in oesophagus cancer, Br. J. Cancer 106 (2012) 1415-1423.

[38] G.S. Huang, J. Brouwer-Visser, M.J. Ramirez, C.H. Kim, T.M. Hebert, J. Lin, et al. Insulin-like growth factor 2 expression modulates Taxol resistance and is a candidate biomarker for reduced disease-free survival in ovarian cancer, Clin. Cancer Res. 16 (2010) 2999-3010.

[39] J. Brouwer-Visser, J. Lee, K. McCullagh, M.J. Cossio, Y. Wang, G.S. Huang, Insulin-like growth factor 2 silencing restores taxol sensitivity in drug resistant ovarian cancer, PLoS One 9 (2014), e100165.
[40] A. Slipicevic, G.F. Oy, I.C. Askildt, A. Holth, E. Hellesylt, V.A. Florenes, et al., Diagnostic and prognostic role of the insulin growth factor pathway members insulin-like growth factor-II and insulin-like growth factor binding protein-3 in serous effusions, Hum. Pathol. 40 (2009) 527-537.

[41] B. Boyerinas, S.M. Park, A. Hau, A.E. Murmann, M.E. Peter, The role of let-7 in cell differentiation and cancer, Endocr. Relat. Cancer 17 (2010) F19-F36.

[42] A. Ruzzo, F. Graziano, B. Vincenzi, E. Canestrari, G. Perrone, N. Galluccio, et al., High let-7a microRNA levels in KRAS-mutated colorectal carcinomas may rescue antiEGFR therapy effects in patients with chemotherapy-refractory metastatic disease, Oncologist 17 (2012) 823-829.

[43] B.X. Yan, J.X. Ma, J. Zhang, Y. Guo, H. Riedel, M.D. Mueller, et al., PSP94 contributes to chemoresistance and its peptide derivative PCK3145 represses tumor growth in ovarian cancer, Oncogene 33 (2014) 5288-5294. 